\title{
Investigating turbulent structure of ionospheric plasma velocity using the Halley SuperDARN radar
}

\author{
G. A. Abel, M. P. Freeman, G. Chisham, and N. W. Watkins \\ British Antarctic Survey, Natural Environment Research Council, High Cross, Madingley Road, Cambridge, CB3 0ET, UK
}

Received: 22 March 2007 - Revised: 16 November 2007 - Accepted: 16 November 2007 - Published: 7 December 2007

\begin{abstract}
We present a detailed analysis of the spatial structure of the ionospheric plasma velocity in the nightside Fregion ionosphere, poleward of the open-closed magnetic field line boundary (OCB), i.e. in regions magnetically connected to the turbulent solar wind. We make use of spatially distributed measurements of the ionospheric plasma velocity made with the Halley Super Dual Auroral Radar Network (SuperDARN) radar between 1996 and 2003. We analyze the spatial structure of the plasma velocity using structure functions and $P(0)$ scaling (where $P(0)$ is the value of the probability density function at 0 ), which provide simple methods for deriving information about the scaling, intermittency and multi-fractal nature of the fluctuations. The structure functions can also be compared to values predicted by different turbulence models. We find that the limited range of velocity that can be measured by the Halley SuperDARN radar restricts our ability to calculate structure functions. We correct for this by using conditioning (removing velocity fluctuations with magnitudes larger than 3 standard deviations from our calculations). The resultant structure functions suggest that Kraichnan-Iroshnikov versions of $P$ and log-normal models of turbulence best describe the velocity structure seen in the ionosphere.
\end{abstract}

\section{Introduction}

Ionospheric convection is driven by the solar wind and regulated by magnetic reconnection on the magnetopause and in the magnetotail. These processes are well understood on the average global scale (e.g., Kamide and Baumjohann, 1993, pp. 17-30) and give rise to a two-cell convection pattern during periods of southward interplanetary magnetic field (IMF), a four-cell convection pattern during periods of strong

Correspondence to: G. A. Abel

(gaab@bas.ac.uk) northward IMF, with modifications of these patterns by the east-west component of the IMF (Ruohoniemi and Greenwald, 2005). However, at scales smaller than the global scale, ionospheric convection is less well understood and has been shown to be complex and structured both temporally (Abel and Freeman, 2002) and spatially (Abel et al., 2006).

Many studies of fluctuations in the MagnetosphereIonosphere (M-I) system have demonstrated the existence of scale-free structure, i.e. a measure of fluctuations with no characteristic scales (power law), over a wide range of scales. Examples include (a) power spectra of ground magnetic fields (Cambell, 1976; Francia et al., 1995; Consolini et al., 1998; Weatherwax et al., 2000; Abel and Freeman, 2002), and ionospheric electric fields (Kintner, 1976; Weimer et al., 1985; Bering et al., 1995; Buchert et al., 1999; Abel and Freeman, 2002; Golovchanskaya et al., 2006), (b) probability density functions (PDFs) of durations between threshold crossings of the auroral electrojet indices AU and AL (Freeman et al., 2000), (c) PDFs of durations, areas, and other quantities of auroral bright patches (Lui et al., 2000; Uritsky et al., 2002; Kozelov et al., 2004) and (d) structure functions of the auroral electrojet indices $\mathrm{AU}, \mathrm{AL}$ and $\mathrm{AE}$, the polar cap index PC (Takalo et al., 1993; Takalo and Timonen, 1998; Hnat et al., 2002), ground magnetic fields (Pulkkinen et al., 2006) and ionospheric convection (Parkinson, 2006; Abel et al., 2006).

The majority of these studies have been performed in the temporal domain (or combined spatial and temporal domains) for which good statistics over a wide range of scales are easier to achieve. For example, an instrument operating continuously for one year at a fixed location with 1-s sampling covers seven orders of magnitude of timescales, with over $10^{7}$ data points at all timescales up to 0.5 years. Such a wide range of scales is much harder to achieve in the spatial domain as it requires simultaneous measurements at a large number of measurement locations. Some studies have inferred spatial structure from time series by assuming

Published by Copernicus Publications on behalf of the European Geosciences Union and the American Geophysical Union. 
that the relative motion of the instrument and the structure it measures is sufficiently fast that temporal variations can be ignored (the Taylor hypothesis (Taylor, 1938)). Whilst this is a commonly used assumption for solar wind data, it is more questionable whether it can be applied to electric and magnetic fields measured by low-altitude spacecraft, as some studies have done (Kintner, 1976; Weimer et al., 1985; Golovchanskaya et al., 2006).

Two recent studies have measured the spatial structure in the ionosphere without making this assumption. Pulkkinen et al. (2006) analyzed ground magnetometer data from the IMAGE network to infer the structure of ionospheric currents in both the spatial and temporal domains. They found evidence for scale-free structure from 100-1000 km, though with only 6 bins in the spatial domain. Abel et al. (2006) analyzed ionospheric velocities measured by the Halley Super Dual Auroral Radar Network (SuperDARN) radar and found evidence for scale-free structure from the spatial resolution of the data $(45 \mathrm{~km})$ to $\sim 1000 \mathrm{~km}$. At scales larger than $1000 \mathrm{~km}$ deviation from scale-free structure was seen, consistent with the global 2-cell convection pattern.

Two mechanisms have been invoked to explain the presence of scale-free structure in the M-I system, namely self-organized criticality (SOC) (Chang, 1992) and magnetohydrodynamic (MHD) turbulence (e.g., Kintner, 1976; Borovsky et al., 1997). It has been hypothesized (Freeman et al., 2000) that the scale-free structure is either internal to the M-I system (e.g. SOC or MHD turbulence in the magnetotail) or inherited from the scale-free structure of the solar wind, which is known to be turbulent. Different scale-free structure has been observed in different regions of the M-I system (Consolini et al., 1998; Takalo and Timonen, 1998; Hnat et al., 2002), indicating that both hypotheses may play a role. In particular, Abel et al. (2006) found evidence for scale-free structure with different scaling exponents in areas of open and closed magnetic field topology, i.e. magnetically connected to, and isolated from, the solar wind, respectively.

In this paper we present a detailed analysis of the spatial structure of the ionospheric F-region plasma velocity using a subset of the data presented by Abel et al. (2006). This subset comprises velocity fluctuations in the nightside ionosphere poleward of the open-closed field line boundary (OCB), this being the region with most data in the original study and magnetically connected to the turbulent solar wind. We analyze the spatial structure of the plasma velocity using structure functions and $P(0)$ scaling, which provide simple methods for deriving information about the scaling, intermittency and multi-fractal nature of the fluctuations. In the solar wind a number of studies have used the results of structure function analysis as evidence for various models of turbulence, e.g. the P model of Kolmogorov turbulence (PK41) and the $\mathrm{G}$ infinity model (Pagel and Balogh, 2001). Here we perform a similar analysis to test the applicability of various models of turbulence to ionospheric plasma velocity structure and compare our findings to previous studies of the solar wind.

\section{Methodology}

In this study we make use of structure function analysis to investigate the structure of ionospheric convection measured by the Halley SuperDARN radar (Greenwald et al., 1995; Chisham et al., 2007). Compared to Fourier and many other analysis techniques that require regularly sampled data, structure function analysis can be applied to data that are patchy in time and space, which makes it suitable for analysing SuperDARN data that have many data gaps.

In standard turbulence analysis the velocity structure functions is defined as (e.g., Frisch, 1995)

$S_{n}(l)=<([v(r+l, t)-v(r, t)] \cdot \hat{l})^{n}>$

where $v$ is the velocity measured at position $r$ and time $t, l$ is the separation between two measurements and $<\cdot>$ denotes the ensemble average. $v, r$ and $l$ are all vector quantities and $\hat{l}$ is a unit vector in the direction of the flow.

However, it has been shown that the scaling exponents calculated from the standard structure functions defined by Eq. (1) can only be measured securely for significantly large Reynolds numbers and also suffer from poor statistical convergence (Grossmann et al., 1997). Furthermore, in the case considered here only one component of the velocity can be measured by the radar and is the same direction as the range separation. This component is in the line of sight (LOS) of the radar beam and thus is parallel to to $\hat{l}$ but may not be in the direction of the flow required by Eq. (1). For these reasons we should not use Eq. (1) to measure ionospheric convection structure.

Instead it has been argued that it is essential to calculate structurefunctions from the moduli of the velocity differences:

$S_{n}(l)=<|v(r+l, t)-v(r, t)|^{n}>$

where $v(r, t)$ is a convection velocity component measured at position $r$ and time $t, l$ is the separation distance between two measurements in the LOS direction. In this case reliable scaling exponents are recovered, that are both independent of Reynolds number and the flow geometry (Grossmann et al., 1997, and references therein)

In a turbulent fluid it is predicted that $S_{n}$ will scale with the separation distance $l$, i.e. $S_{n}(l) \sim l^{\zeta_{n}}$ where $\zeta_{n}$ is the scaling exponent of the $n$th order structure function. If the energy transfer rates between scales are homogeneous then $\zeta_{n}=n \zeta_{1}$. Otherwise the turbulence is termed intermittent and $\zeta_{n}$ has a more complicated relationship with $n$ (e.g., Bohr et al., 1998, pp. 31-43). In Appendix A we list the $\zeta_{n}$ equations for a number of models of turbulence. It is worth noting that we have calculated odd order structure functions without taking the absolute values of the velocity fluctuations (not shown) and do not find the scaling structure shown below. We believe that this is due to the dominance of the global scale convection pattern. 
The convection velocity component $v$ is measured by the Halley SuperDARN radar. SuperDARN radars measure backscatter from magnetic field-aligned density irregularities in the $\mathrm{E}$ and $\mathrm{F}$ regions of the ionosphere. The radars transmit at fixed frequencies in the $8-20 \mathrm{MHz}$ range and, from the return signals, estimates can be made of backscatter power, line-of-sight (LOS) Doppler velocity, and Doppler spectral width. The SuperDARN radars operate for much of the time in a common mode, in which the radars scan through 16 beam directions in either 60 or $120 \mathrm{~s}$, beam centers are separated by $\sim 3.25^{\circ}$ in azimuth with a beam width of $\sim 5^{\circ}$, and along each beam 75 range gates are measured at $45 \mathrm{~km}$ separation (equivalent to a pulse length of $300 \mu \mathrm{s}$ ) from a first range at $180 \mathrm{~km}$ (equivalent to a lag to the first range of $1200 \mu \mathrm{s}$ ). In this mode, the SuperDARN radars can measure LOS velocities up to about $\pm 2000 \mathrm{~m} \mathrm{~s}^{-1}$, the limit varying slightly depending on the operational frequency. Velocities outside this range will be aliased.

In this study we use only common mode data measured by the Halley SuperDARN radar during the 8-year interval 1996-2003 inclusive. The velocity differences are assumed to be stationary over this interval (e.g. no significant seasonal or long term trends). Future studies will test the strength of this assumption by considering long term effects such as solar cycle dependence, though it should be noted that due to radar scatter statistics the majority of measurements used in this study were made close to solar maximum (in 2000 and 2001). In addition, we only use data from the beam aligned along the geomagnetic meridian (beam number 8 ) so as not to complicate our analysis by combining data from different look directions. We further restrict ourselves to data taken at range gate 10 and higher so as to retain only F-region backscatter for which the LOS velocity is a reliable estimate of the plasma $E \times B$ drift velocity (Villain et al., 1985; Ruohoniemi et al., 1987). Finally, we also restrict the data to a subset of that used by Abel et al. (2006) corresponding to the region of open magnetic field lines in the nightside ionosphere (18-02 MLT). We do this because the first-order structure function was found to be different in the open and closed field line regions and the nightside open field line region had the largest amount of data. The OCB location was estimated using the C-F spectral width boundary method (Chisham and Freeman, 2003, 2004) and we restrict the analysis to those magnetic local times (MLTs) where, statistically, the spectral width boundary is known to match well to the OCB determined independently from polar-orbiting satellite measurements of charged particle precipitation in the nightside ionosphere (Chisham et al., 2004, 2005).

After applying these restrictions, the analysis algorithm is as follows: For each radar scan for which the OCB could be identified, we select all pairs of LOS velocity measurements poleward of the OCB for a given separation $l$ (where $l$ is an integer multiple of the $45 \mathrm{~km}$ range gate separation) and subtract the more equatorward measurement of LOS velocity from the more poleward one to

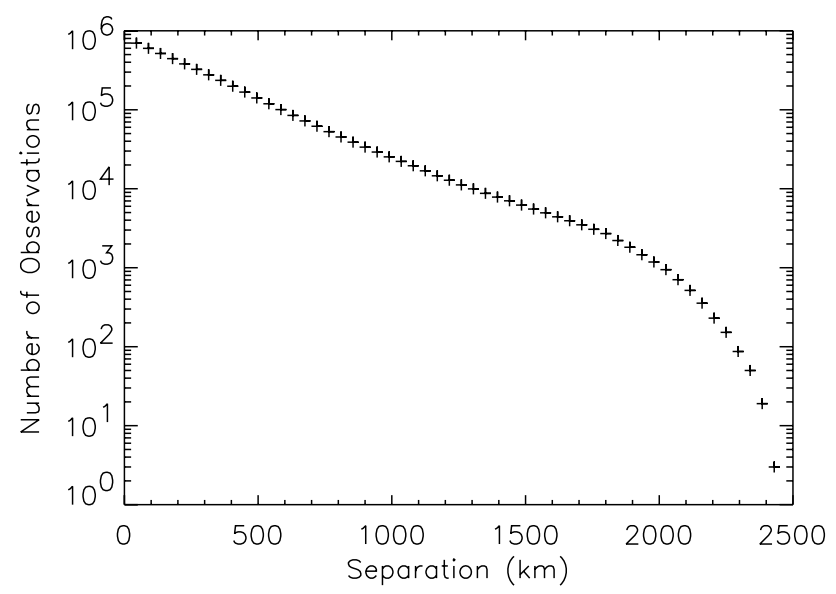

Fig. 1. The number of $\Delta v$ measurements as a function of separation $l$ used in the calculation of $S$ for the unconditioned data.

give $\Delta v(l)=v(r+l, t)-v(r, t)$. We then take the moduli of these values and average over all scans from the 8-year period $(<|v(r+l, t)-v(r, t)|>)$. This is then repeated for all possible range gate separations from $1(l=45 \mathrm{~km})$ to 55 $(l=2475 \mathrm{~km})$ to give $S_{1}(l)$. Similar calculations are performed for the second, third, fourth, fifth and sixth order structure functions $(n=2,3,4,5$, and 6 respectively). At the same time we calculate PDFs of the velocity differences $\Delta v$ for each separation $l$, in bins of $10 \mathrm{~m} \mathrm{~s}^{-1}$.

\section{Analysis of raw data}

Figure 1 shows the occurrence frequency of $\Delta v$ measurements as a function of separation $l$ when the selection criteria described above are applied to the Halley SuperDARN data. There are many more data pairs at small separations than at larger ones. This is a result of two factors: (1) At any one time SuperDARN backscatter is only measured at a limited (but variable) number of ranges. (2) The fact that we are considering only measurements made poleward of the OCB restricts the number of large separations. At a separation of 1 range gate $(45 \mathrm{~km})$ we have $>10^{5}$ pairs of measurements. At $2000 \mathrm{~km}$ separation this falls to below $10^{3}$ data pairs.

Figure 2 shows the first three order structure functions plotted as a function of separation calculated using the algorithm presented above. It should be noted that $S_{2}$ has been divided by 200 and $S_{3}$ has been divided by 50000 in order to show them clearly on the same figure as $S_{1}$ (it is the shape and slope of the line that we are most interested in, rather than the absolute value). For each of the three lines we see a similar form with a power-law (i.e. straight line on a log-log plot) at small separations $(<\sim 600-1000 \mathrm{~km})$ and a deviation from this at large separations $(>\sim 600-1000 \mathrm{~km})$. The deviation from power-law behavior seen at large separations was attributed by Abel et al. (2006) to the global 2-cell convection pattern. 


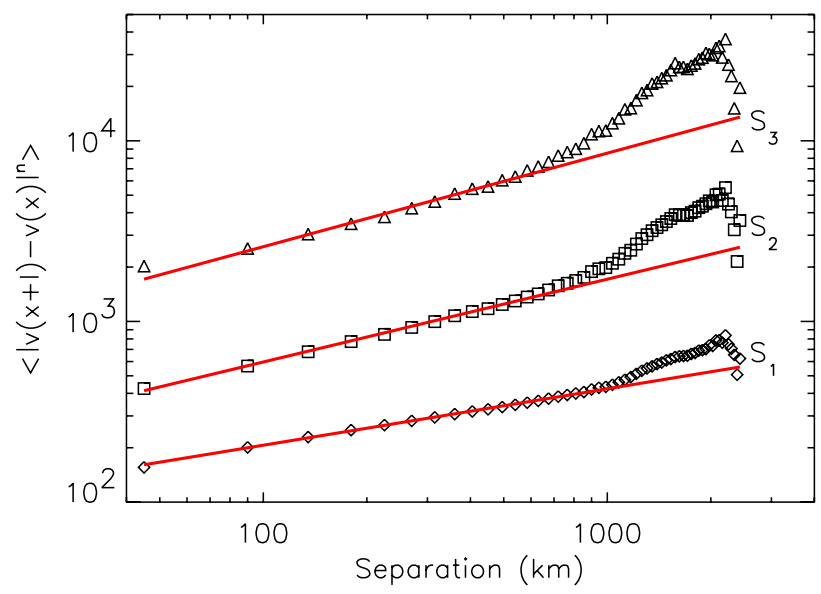

Fig. 2. The first three order structure functions plotted as a function of separation $l$ calculated using unconditioned data ( $S_{1}$ diamonds, $S_{2}$ squares, $S_{3}$ triangles). For convenience $S_{2}$ has been divided by 200 and $S_{3}$ has been divided by 50000 . The red lines show straight lines fitted to the power-law region of each structure function.

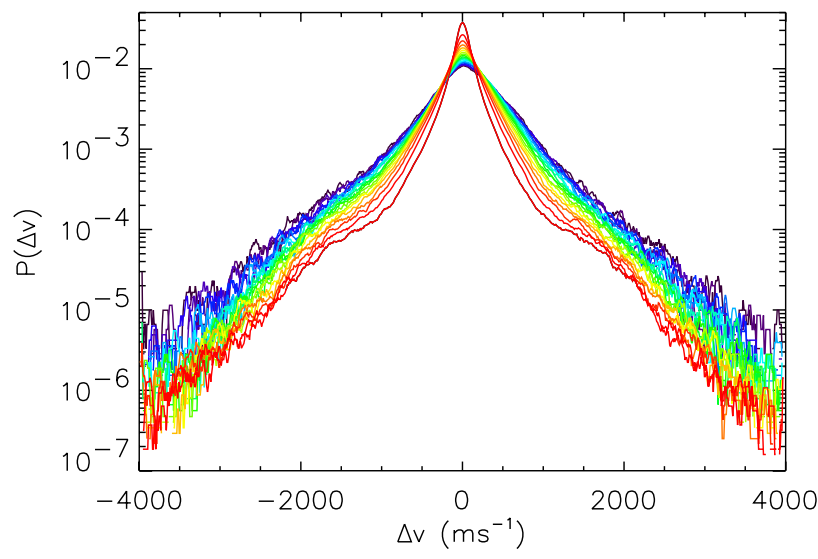

Fig. 3. PDFs of $\Delta v$ used in the calculation of structure functions for separations from 1 (red) to 20 (black) range cells (45-900 km). The data is plotted for $10 \mathrm{~m} \mathrm{~s}^{-1}$ bins with a 9-point running mean applied.

The red lines in Fig. 2 show straight lines fitted to powerlaw regions of each line (using a least-squares fit to the points in log-log space, where the extent of the fit is selected by eye). The slope of the line in log-log space is the power-law exponent $\zeta_{n}$. The fitted power-law exponents are $\zeta_{1}=0.31$ (from 135 to $945 \mathrm{~km}$ ), $\zeta_{2}=0.48$ (from 45 to $675 \mathrm{~km}$ ) and $\zeta_{3}=0.52$ (from 90 to $585 \mathrm{~km}$ ). In order to asses the sensitivity of our estimates of $\zeta_{n}$ to the fitting range we have also fitted the lines over half the number of data points. None of the fitted exponents presented in this paper change by more than 0.01 .

In order to assess the validity of our calculated structure functions and their scaling exponents we study the PDFs of the velocity fluctuations used in our calculations. These are shown in Fig. 3 for separations of 1 (red) to 20 (black) range cells $(45-900 \mathrm{~km})$. The data are plotted using $10 \mathrm{~m} \mathrm{~s}^{-1}$ bins with a 9-point running mean applied. The PDFs reveal two possible sources of error. Firstly, statistical fluctuations increase with increasing $\Delta v$ and increasing $l$ due to a decreasing number of data samples. As the PDFs are non-Gaussian leptokurtic distributions the tails of the distributions with larger statistical fluctuation error contribute significantly to the calculated structure functions (increasingly so for increasing $n$ ). Secondly, at around $\pm 2000 \mathrm{~m} \mathrm{~s}^{-1}$ there is a shoulder in the PDFs (most clearly seen in the red trace). This does not reflect the actual velocity fluctuations in the ionosphere but is due to the maximum velocity that can be measured by the radar. In common mode, SuperDARN velocity measurements are aliased outside of the range $\left|v_{\max }\right| \approx 2000 \mathrm{~m} \mathrm{~s}^{-1}$ (which varies slightly depending on the operational frequency of the radar). Hence, the maximum velocity difference that can be measured is $\Delta v=2 v_{\max }$. Given that some measurements of $\Delta v$ will have been calculated when $v(r+l, t)$ or $v(r, t)$ (or both) have been aliased, the measured PDF of $\Delta v$ will be different from the true PDF of the system. This effect will be most significant when $\Delta v$ is close to or greater than $v_{\max }$ and we believe this effect gives rise to the shoulder seen in Fig. 3 at around $\pm 2000 \mathrm{~m} \mathrm{~s}^{-1}$. It should be remembered that only a small number of velocity measurements will be aliased but due to the heavy tailed nature of the $\Delta v$ PDF it will have a significant effect when calculating $S_{n}$. The effect of aliasing on the PDF at small $\Delta v$ will be insignificant and the central core of the distribution is estimated well with low statistical fluctuation error.

The poor estimate of the PDF at large $\Delta v$ will adversely affect the calculated structure functions shown in Fig. 2 and hence the scaling exponents found. The point at which this effect becomes significant is at a fixed velocity difference $\left(\approx v_{\max }\right)$ and not at a fixed percentile of the distribution. To tackle this problem and determine more accurate structure functions we need to remove the erroneous data at large $\Delta v$ whilst retaining the same proportion of the distribution at each separation $l$.

\section{Analysis of conditioned data}

To correct for the sources of error described above we have applied a technique called conditioning. This removes data that is possibly erroneous by clipping the data used in the structure function calculations so that all fluctuations larger than $b \sigma_{\Delta v}(l)$ are ignored, where $b$ is a constant and $\sigma_{\Delta v}(l)$ is the standard deviation of the velocity differences at range separation $l$. Applying the conditioning technique ensures that the same proportion of the parent distribution for each $l$ is retained (and also that the calculation of $S_{n}$ is based on data drawn from a distribution with finite moments). Chapman et al. (2005) showed that the heavy-tailed distributions of a 
modeled Lévy flight time series made calculations of structure functions difficult because the moments are strongly influenced by the tails of the distributions, which are poorly sampled statistically (i.e. rare). They demonstrated that by removing the poorly sampled data using conditioning, the known mono-fractal scaling behavior could be restored, i.e. $\zeta_{n}=n \zeta_{1}$. The same technique has also been applied to the $\mathrm{AE}$ index (Chapman et al., 2005) and measurements of the solar wind (Hnat et al., 2003, 2005). Furthermore, a similar technique has been applied in wavelet spectral techniques in studies of atmospheric turbulence (i.e., Katul et al., 1994) and in the solar wind (Veltri and Mangeney et al., 1999; Bruno et al., 1999; Mangeney et al., 2001). Clearly using a conditioned data-set will not give a good absolute estimate of $S_{n}$. What we are really interested in is the scaling properties of $S_{n}$ (i.e. $\zeta_{n}$ ), which may be based on part of the distributions.

In their study, Chapman et al. (2005) clipped their data at $b=5,10,15$ and 20. However in our case we need to clip at a smaller $b$ because we wish to remove not only the rarely sampled data but also the erroneous data that results from velocity aliasing. The effect of the aliasing is to intro-

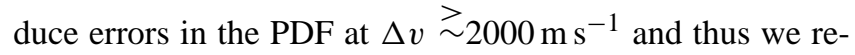
quire $b \sigma_{\Delta v}<2000 \mathrm{~m} \mathrm{~s}^{-1}$. However, we do not need to satisfy this criteria for the whole data set as we are only concerned with a correct application of conditioning at the separations where scaling is seen (i.e. $l<900 \mathrm{~km}$ ). As $\sigma_{\Delta v}$ increases with $l$ the largest $b \sigma_{\Delta v}$ we are concerned with occurs when $l=900 \mathrm{~km}$. At this separation $\sigma_{\Delta v}=616 \mathrm{~m} \mathrm{~s}^{-1}$, and so we have chosen to clip our data at $b=3$ (i.e. $b \sigma_{\Delta v}=1848 \mathrm{~m} \mathrm{~s}^{-1}$ ). In fact, clipping our data at $3 \sigma_{\Delta v}$ ensures that fluctuations $> \pm 2000 \mathrm{~m} \mathrm{~s}^{-1}$ are not used in the calculation of $S_{n}$ for separations of $1035 \mathrm{~km}$ or less. By clipping at $b=3$ there is a $2 \%$ reduction in data overall compared to the unconditioned calculations. Proportionately most data is lost at small separations with a $2.1 \%$ reduction in data at $45 \mathrm{~km}$ separation and a $1.8 \%$ reduction in data at $900 \mathrm{~km}$ separation.

To understand better how the clipping affects the calculation of $S_{n}$, and hence $\zeta_{n}$, and which order of structure functions we can trust, we investigate the partial distribution of $S_{n}$ as a function of $\Delta v$. Figure 4 presents histograms showing the proportion of $S_{n}$ to come from each $100 \mathrm{~m} \mathrm{~s}^{-1} \Delta v$ bin for $n=1,2,3,4,5,6$. Each panel in Fig. 4 shows the histograms for different separations from $45 \mathrm{~km}$ (a) to $900 \mathrm{~km}$ (d). The grey vertical lines in each panel show the $3 \sigma_{\Delta v}$ clipping that has been applied i.e. we ignore all data to the right of the grey line.

In each panel of Fig. 4 we see that the peaks of the distributions are at small $\Delta v$ for $S_{1}$, moving to increasing values with increasing order. For the larger separations (panels c and d) the peak is hard to see for the higher order curves due to the statistical fluctuations. These fluctuations are due to poor sampling statistics and the emphasis of larger $\Delta v$ for larger moments. It is clear that by clipping at $3 \sigma_{\Delta v}$ we ignore most of these poorly sampled bins. More importantly,
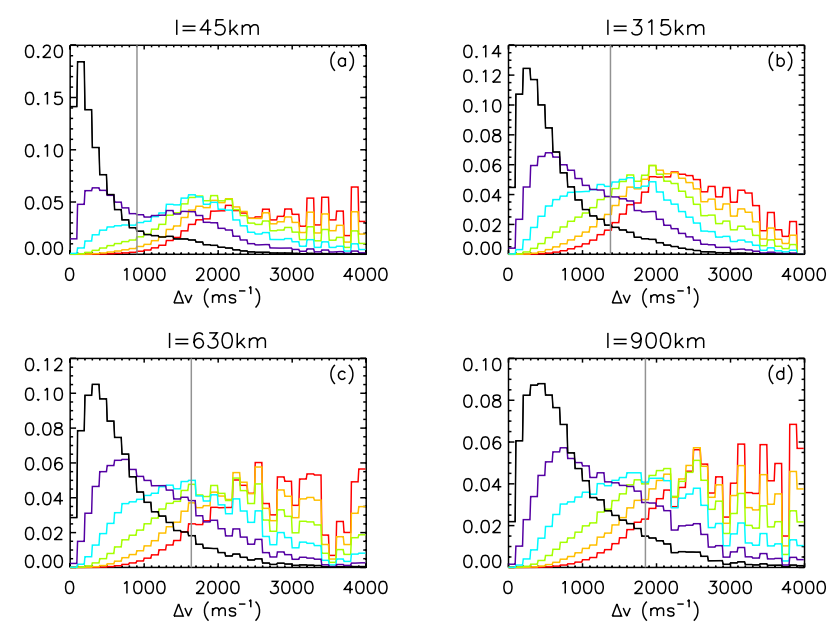

Fig. 4. Partial distributions showing the proportion of $S_{n}$ to come from each $100 \mathrm{~m} \mathrm{~s}^{-1} \Delta v$ bin. Each panel shows the partial distribution for a different separation; (a) $l=45 \mathrm{~km}$, (b) $l=315 \mathrm{~km}$, (c) $l=630 \mathrm{~km}$ and (d) $l=900 \mathrm{~km}$. Each panel shows the partial distributions for the first six order structure functions; $n=1$ (black), $n=2$ (purple), $n=3$ (blue), $n=4$ (green), $n=5$ (yellow), and $n=6$ (red).

if we look at the partial distribution of $S_{1}$ (black line) we see that for all separations the range of data included in our calculations (left of the grey line) includes most of the distribution and certainly includes its peak and form. We would say that this is also true of $S_{2}$ (purple line) especially when we consider that the secondary peak seen in panel (a) between 1100 and $2000 \mathrm{~m} \mathrm{~s}^{-1}$ is due to the aliasing problem that we are attempting to exclude. In the case of $S_{3}$ it is much more marginal but we certainly would not trust $S_{4}, S_{5}$ or $S_{6}$, as our calculation only includes the lower tail of the partial distribution and hence we would not expect an accurate estimate of $\zeta_{n}$ from this.

Figure 5 shows the conditioned structure functions plotted as a function of separation for the first three orders. It should be noted that $S_{2}$ has been divided by 200 and $S_{3}$ has been divided by 50000 as in Fig. 2. The same shape of line is seen in Fig. 5 as in Fig. 2 with power-law behavior seen at small separations and deviations due to the global convection cells seen at large separations. The red lines in Fig. 5 show straight lines fitted to power-law regions of each line (using a least-squares fit to the points in log-log space where the extent of the fit is selected by eye). The fitted power-law exponents are $\zeta_{1}=0.34$ (from 135 to $945 \mathrm{~km}$ ), $\zeta_{2}=0.63$ (from 45 to $675 \mathrm{~km}$ ) and $\zeta_{3}=0.88$ (from 90 to $585 \mathrm{~km}$ ).

We have also tried conditioning at different $b$ between 1 and 9. The result of this different clipping is shown in Fig. 6. The clipping makes little difference to the value of $\zeta_{1}$ because the tails of the $\Delta v$ PDF make little contribution to $S_{1}$. For $n=2$ and $n=3$ we see a considerable change in $\zeta_{n}$ with $b$ when $b>4$ indicating that such levels of clipping are not successful in removing eroneous data (as to be expected given 


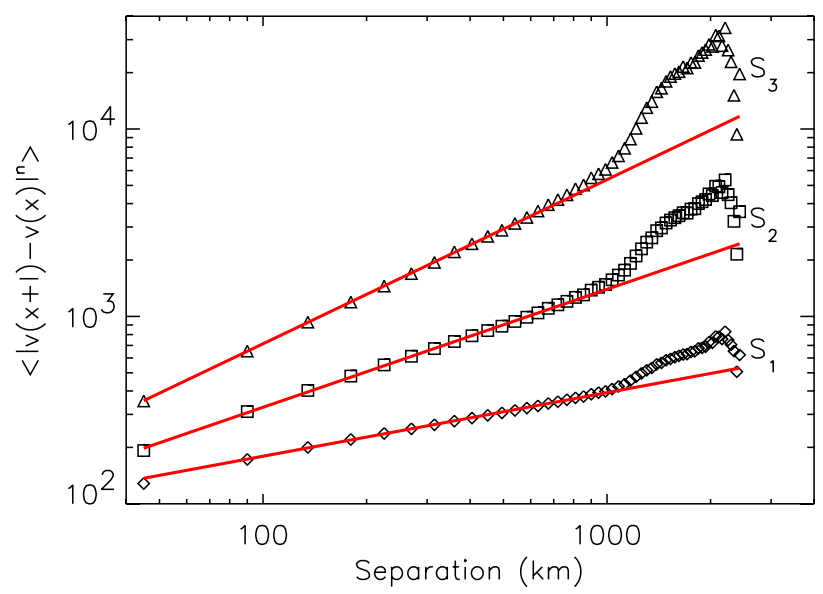

Fig. 5. The first three order structure functions plotted as a function of separation $l$ calculated using conditioned data ( $S_{1}$ diamonds, $S_{2}$ squares, $S_{3}$ triangles). For convenience $S_{2}$ has been divided by 200 and $S_{3}$ has been divided by 50000 . The red lines show straight lines fitted to the power-law region of each structure function.

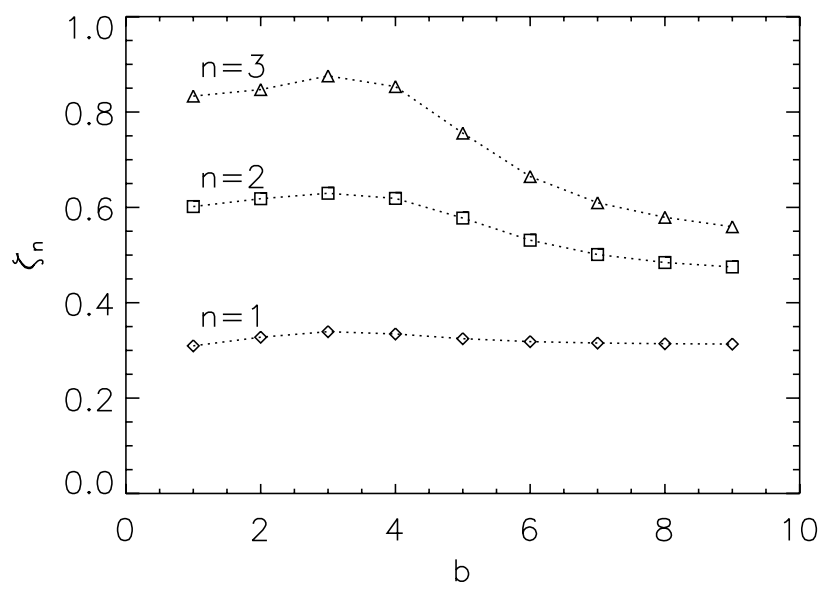

Fig. 6. The variation of the fitted power-law exponents $\zeta_{n}$ for different levels of clipping $b$ for the first three order structure functions.

the arguments above). When $b \leq 3$, reasonably similar results give us confidence in the robustness of the results presented above (Fig. 5). There is some small variation for $b \leq 3$ which will occur due to sampling less of the core of the partial distributions shown in Fig. 4.

\section{$5 \quad P(0)$ scaling}

In addition to the structure functions shown above, further information can be gleaned about the structure of the ionospheric plasma velocities by calculating the peak, or $P(0)$, scaling. Figure 7 shows the peak values of the PDFs of ionospheric velocity fluctuations as a function of separation. Here we see a power law region from $\sim 135 \mathrm{~km}$ to over

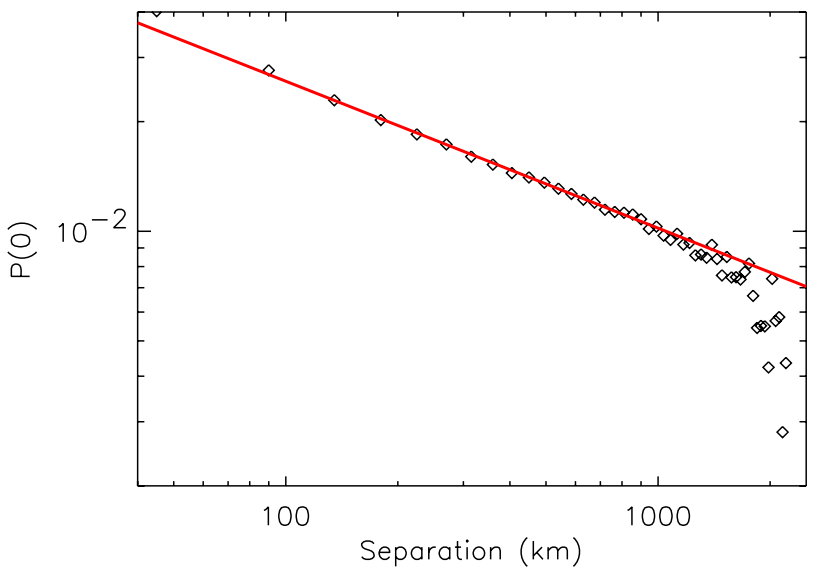

Fig. 7. Peaks of the PDFs of $\Delta v$ as a function of separation $l$ (diamonds). The red line shows a straight line fitted to the powerlaw region of the $P(0)$ line.

$1000 \mathrm{~km}$. The red line in Fig. 7 shows a straight line fitted over the range $135-945 \mathrm{~km}$ (again using a least-squares fit to the line in log-log space where the extent of the fit is selected by eye). The fitted power-law exponent is -0.40 . It is worth noting that the $P(0)$ scaling is not affected by the same issues that made calculating the structure functions difficult. The peaks of our PDFs always occur close to zero and so they are not significantly affected by the aliasing problem discussed above. Moreover, they are calculated from the region with the best statistics, i.e. the part of the distribution we capture best.

\section{Discussion}

\subsection{Comparison with turbulence models}

Figure 8 shows $\zeta_{n}$ as a function of $n$ for the first three order structure functions (black and red points), and compares these measured values against eight different models of turbulence (red lines). The models shown are the "classic" (nonintermittent) Kolmogorov (K41) and Kraichnan-Iroshnikov (KI65) models along with Kolmogorov and Kraichnan versions of the intermittent $P, \log$-normal, and G-infinity models. The equations used to determine these model lines are given in the appendix along with the values of any free parameters used. The non-intermittent K41 and KI65 models have no free parameters. All other models have one free parameter which has been determined using $\zeta_{1}$, the best defined moment. The red points indicate those which have been used to determine the free parameters. We have also determined the free parameters using a least squares fit to all three points (not shown) and very similar values are found. The error bars shown on the points have been calculated from the variation of $\zeta_{n}$ when conditioning between $b=2$ and $b=4$. Other sources of error are the error associated with the 


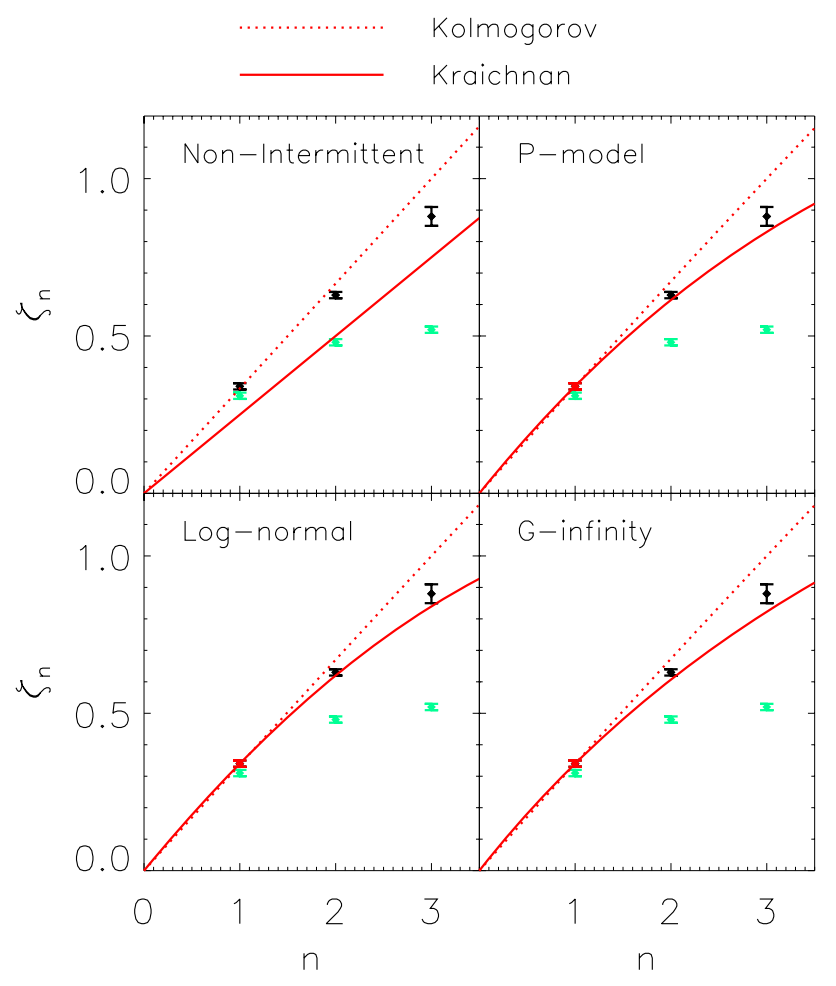

Fig. 8. $S_{1}, S_{2}$ and $S_{3}$ calculated from the conditioned plasma velocity data (black and red points) compared to eight different turbulence models. In each panel Kolmogorov (dotted line) and Kraichnan (solid line) versions are plotted. Details of the $\zeta_{n}$ vs. $n$ relationships can be found in the appendix along with the values of the free parameters used. The points plotted in red have been used to derive free parameters and so will give perfect agreement with the plotted curves. Points plotted in black do not constrain the plotted curves in any way. Also shown are the values of $S_{1}, S_{2}$ and $S_{3}$ calculated from the unconditioned plasma velocity data (green points).

selection of the region of fit $(\sim 0.1)$ and the error on the least squares fit $(\sim 0.003)$ neither of which will affect the error bars shown in Fig. 8.

At the most conservative level we consider that only $\zeta_{1}$ is wholly trustworthy. In this case, we cannot judge the goodness of fit of the intermittent models as the free parameters have been determined using $\zeta_{1}$. However, it is interesting to note that all of the Kolmogorov-type intermittent models are very close to the non-intermittent version. This is because $\zeta_{1}$ is very close to the value of $1 / 3$ predicted for K 41 . Conversely the non-intermittent KI65 does not agree with $\zeta_{1}$ and the fitted Kraichnan-type models all show significant intermittency. Let us now take a more liberal view of what moments we can trust and consider $\zeta_{1}$ and $\zeta_{2}$ and possibly $\zeta_{3}$. The Kolmogorov-type models all give good agreement with $\zeta_{1}$ and $\zeta_{2}$ but not $\zeta_{3}$. The intermittent Kraichnan models give good agreement with $\zeta_{2}$ (and $\zeta_{1}$ by construction) and a reasonable agreement with $\zeta_{3}$ considering the uncertainty of this value. The Kraichnan versions of the log-normal model gives the best agreement with our data followed by the $\mathrm{P}$ model and then the G-infinity model. It should be noted that all Kolmogorov-based models are constrained such that $\zeta_{3}=1$ which does not agree with our measured $\zeta_{3}$ value.

One might argue that it is not fair to compare $\zeta$ values calculated from our conditioned data to the analytical predicted values for different turbulence models in the absence of conditioning. By removing large fluctuations from our calculations we are removing some of the intermittency which gives rise to the $\zeta$ vs $n$ relationships predicted for intermittent models of turbulence (such as the $P$-models shown here) and thus we might expect different $\zeta$ vs $n$ relationships for conditioned data. It is for precisely this reason that we have chosen to ignore $\zeta_{n}$ when $n \geq 4$. For $\zeta_{1}$ we argue that the amount of the partial distribution of $S_{1}$ that is included in our calculations is enough that we represent the true value of $\zeta_{1}$ well, or conversely that the expected value of $\zeta_{1}$ from a model will not change significantly when the model is subjected to conditioning. As we have explained above, the argument is less strong for $\zeta_{2}$ and marginal for $\zeta_{3}$.

Based on structure functions alone, and knowing that $\zeta_{3}$ is questionable, it is hard to argue that any one model shown in Fig. 8 better agrees with our data than any other (except for dismissing the non-intermittent KI65). However, we can use the fact that we know $P(0)$ has a scaling exponent of 0.40 to add more information. As we mentioned above the fitted Kolmogorov-type models were all close to the nonintermittent version. The non-intermittent K41 model is a mono-fractal, i.e. it has only one scaling exponent and $\zeta_{n}=n \zeta_{1}$. If our data were described by a mono-fractal we would expect $P(0)$ to scale with the same exponent $\zeta_{1}$. The reason for this is trivial when considering Guassian fluctuations with zero mean where $P(0)=1 / \sqrt{2 \pi} \sigma$ and the standard deviation, $\sigma$, is equal to $\sqrt{S_{2}}$ and scales with the exponent $\zeta_{2} / 2=\zeta_{1}$. More generally if $v$ were self affine then

$P(\Delta v, l)=l^{-\zeta_{1}} \phi\left(\frac{\Delta v}{l \zeta_{1}}\right)$

(e.g., Krishnamurthy et al., 2000) hence $P(\Delta v=0, l)$ scales as $l^{-\zeta_{1}}$.

The fact $P(0)$ scales differently to $\zeta_{1}$ indicates that the system is a multi-fractal and therefore supports an intermittent model over a non-intermittent one. Based on this extra information we suggest that the Kraichnan versions of the $\mathrm{P}$ and log-normal models give better agreement with our data than K41.

\subsection{The validity of conditioning}

There has been some concern expressed to us about the effects of conditioning data collected from a multi-fractal intermittent system. The concern is that intermittency implies the presence of large non-Gaussian fluctuations and that by removing large fluctuations by conditioning the measurement of intermittency is lost. It has been argued previously that 
by conditioning data taken from an intermittent turbulent medium (e.g. atmospheric turbulence, Katul et al., 1994, and solar wind turbulence, Mangeney et al., 2001), the monofractal component is extracted and any signature of multifractality is lost. Moreover, this mono-fractal component relates directly to non-intermittent turbulence models, such as $\mathrm{K} 41$, in the inertial range. In this section we discuss how our data may be interpreted given these concerns.

Firstly, we consider how one might interpret our data if we did not employ conditioning. The rationale for doing this would be along the lines that, although the aliasing effect of the radar does remove some large fluctuations from our data set and include some spurious ones, it still includes all of the large fluctuations measured and so would give the best estimate of the intermittent nature of the data. For comparison the values of $\zeta_{n}$ for the unconditioned data are shown in Fig. 8 as green points. The error bars of \pm 0.01 are estimated from the variation in $\zeta_{n}$ when fitting over different ranges of $\Delta v$. The unconditioned $\zeta_{n}$ do indeed show strong intermittent behavior, as indicated by the deviation from a linear relationship in Fig. 8. However, it is very hard to interpret these data in terms of current turbulence models. All intermittent models of turbulence we have tested against in this paper (along with any others we are aware of) are constrained in two ways. 1) $\zeta_{3}=1$ for Kolmogorov type turbulence (a direct consequence of the four-fifths law (e.g., Frisch, 1995, pp 76 and 133)) and $\zeta_{4}=1$ in the Kraichnan formalism, and 2) $\zeta_{n}>n / 3$ for $n<3$ in the Kolmogorov cases (e.g., Frisch, 1995, pp 133) and $\zeta_{n}>n / 4$ for $n<4$ in the Kraichnan cases. As can be seen in Fig. 8, our unconditioned $\zeta_{n}$ do not meet these constraints. If one was to consider the unconditioned data as the best estimate of intermittency then it can not be described by current turbulence models and a different physically motivated multi-fractal model and/or theory would have to be found. It is interesting to note that the unconditioned structure functions calculated by Mangeney et al. (2001) using solar wind data do not meet these conditions either.

Secondly, we consider the implications if one considers that strong conditioning, such as we have employed here, extracts the mono-fractal turbulent component. In Sect. 4 we argued that our $\zeta_{n}$ values for $n>3$ could not be trusted and $\zeta_{3}$ was questionable. However, these arguments were made based on our ability to measure intermittency. If we now consider that our conditioning does extract the mono-fractal component, and that we can ignore intermittency altogether, there is no longer any reason to doubt the validity of $\zeta_{3}$ or indeed any higher $\zeta_{n}$ values. Looking at the conditioned data in Fig. 8 we see that $\zeta_{1}$ and $\zeta_{2}$ are consistent with the nonintermittent Kolmogorov model but $\zeta_{3}$ is not. The $\zeta_{n}$ in Fig. 8 are close to having a linear relationship with $n$ as might be expected for a mono-fractal but such a line would not pass through the origin by definition. We conclude from this that strong conditioning does not extract purely the mono-fractal component. Furthermore, if we consider other values of $\zeta_{n}$ conditioned at $3 \sigma_{\Delta v}\left(\zeta_{4}=1.09, \zeta_{5}=1.33, \zeta_{6}=1.57\right)$ the case for a mono-fractal is harder to make.

The final thing we consider is the possibility that by conditioning our data we are removing contributions to intermittency from large fluctuations and that our $\zeta_{n}$ values are less intermittent than the real turbulent reality. This is almost certainly true, but what really matters is how significant is the error introduced is. In Sect. 4 we showed using Fig. 4 that the main contribution to $\zeta_{1}$ came from small values of $\Delta v$ (generally $<1.5 \sigma_{\Delta v}$ ) and that the main contribution to $\zeta_{2}$ came from slightly larger values of $\Delta v$ (generally between 0.5 and $2.5 \sigma_{\Delta v}$ ). There may be contributions to $\zeta_{1}$ and $\zeta_{2}$ coming from higher values of $\Delta v$ which were ignored due to the aliasing effect or conditioning (or both) but given the amount of data lost due to conditioning $(\sim 2 \%)$ these contributions would have to occur at a very large $\Delta v$ indeed to be significant.

If we look at the three curves shown in Fig. 6 we see that for $\zeta_{1}, \zeta_{2}$ and $\zeta_{3}$ there is a peak in their value when conditioning at $3 \sigma_{\Delta v}$. When we consider the constraints outlined above for intermittent turbulence models we find that conditioning at $3 \sigma_{\Delta v}$ results in the most intermittent estimates of $\zeta_{n}$ consistent with turbulence theory.

\section{Summary and conclusions}

In this paper we have presented a detailed structure function analysis of the ionospheric plasma velocity in the nightside ionosphere, poleward of the OCB, as measured by the Halley SuperDARN radar. We have found that the maximum velocity that can be measured by the SuperDARN radars restricts our ability to accurately calculate structure functions. However, we correct for this effect by conditioning our data before calculating the structure functions such that fluctuations $>3 \sigma_{\Delta v}$ are removed. By studying the partial distributions of the structure functions as a function of $\Delta v$, we suggest that structure functions of order 3 and less may be used. The scaling exponents found as a result of this, along with the $\mathrm{P}(0)$ scaling exponent, suggest that the Kraichnan versions of either the $\mathrm{P}$ or log-normal model of turbulence best describes the velocity structure seen in the ionosphere, but to distinguish between these would require accurate determinations of $\zeta_{n}$ for $n>3$.

Turbulence based on the KI65 formalism is reasonable because it describes MHD turbulence in a region of strong background magnetic field which is appropriate to the ionosphere permeated by the strong geomagnetic field. Intermittency could also be expected because the region of the ionosphere we are investigating is directly coupled to the solar wind where intermittency has been found. It is interesting to note that the strength of intermittency found in the ionosphere is similar to that found in the solar wind (compare our value of $p=0.854$ with $p \approx 0.8$ found by Pagel and Balogh, 2001). This provides new information on how these regions are coupled and raises the general question of how turbulence 
changes in coupled environments with different symmetries in the equations which describe these environments.

\section{Addendum}

During the reviewing process of this paper certain concerns were raised questioning the validity of the analysis presented here. These concerns relate to the conditioning technique that we have employed. It was suggested that this technique, by removing the largest fluctuations, reduces the intermittency that is measured. As a consequence, it was suggested that the unconditioned data provides a better estimate of the intermittency in the ionospheric velocity fluctuations than the conditioned data. Our view is that this is not the case and that the best estimate of the intermittency for this data set is provided by the conditioned data for the reasons presented in the paper.

\section{Appendix A}

\section{Turbulence models}

Below are the equations for $\zeta_{n}$ for the different models of turbulence shown in Fig. 8 along with the values used for any parameters. See Pagel and Balogh (2001) and references therein for further details. The G-infinity model is an intermittency model rather than a turbulence model but can be adapted for turbulence by introducing the Kolmogorov or Kraichnan constraint that $\zeta_{3}=1$ or $\zeta_{4}=1$, respectively. Similarly, we have adapted the parameters in the log-normal model to meet the constraint $\zeta_{4}=1$ in the Kraichnan version.

K41

$\zeta_{n}=n / 3$

KI65

$\zeta_{n}=n / 4$

$\mathrm{P}$ model of K41

$\zeta_{n}=1-\log _{2}\left(p^{n / 3}+(1-p)^{n / 3}\right)$

where $p=0.601$.

P model of KI65

$\zeta_{n}=1-\log _{2}\left(p^{n / 4}+(1-p)^{n / 4}\right)$

where $p=0.854$.

Lognormal - Kolmogorov version

$\zeta_{n}=\frac{n}{3}+\frac{\mu}{18}\left(3 n-n^{2}\right)$

where $\mu=0.03$

Lognormal - Kraichnan version

$\zeta_{n}=\frac{n}{4}+x\left(4 n-n^{2}\right)$ where $x=0.03$

G-infinity - Kolmogorov version

$\zeta_{n}=\frac{g(\infty) n}{3 g(\infty)-3+n}$

Where $g(\infty)=34.0$.

G-infinity - Kraichnan version

$\zeta_{n}=\frac{g(\infty) n}{4 g(\infty)-4+n}$

Where $g(\infty)=2.83$.

Acknowledgements. We would like to thank G. King, S. Chapman, K. Kiyani and R. Woodard for useful discussions.

Edited by: T. Chang

Reviewed by: three anonymous referees

\section{References}

Abel, G. A., and Freeman, M. P.: A statistical analysis of ionospheric velocity and magnetic field power spectra at the time of pulsed ionospheric flows, J. Geophys. Res., 107, 1470, doi:10.1029/2002JA009402, 2002.

Abel, G. A., Freeman, M. P., and G. Chisham: Spatial structure of ionospheric convection velocities in regions of open and closed magnetic field topology, Geophys. Res. Lett., 33, L24103, doi:10.1029/2006GL027919, 2006.

Bering, E. A., III, Benbrook, J. R., Byrne, G. J., Liao, B., Theal, J. R., Lanzerotti, L. J., and Maclennan, C. G.: Balloon measurements above the South Pole: Study of ionospheric transmission of ULF waves, J. Geophys. Res., 100, 7807-7819, 1995.

Bohr, T., Jensen, M. H., Paladin, G., and Vulpiani, A.: Dynamical systems approach to turbulence, Cambridge University Press, UK, 1998.

Borovsky, J. E., Elphic, R. C., Funsten, H. O., and Thomsen, M. F.: The Earth's plasma sheet as a laboratory for flow turbulence in high- $\beta$ MHD, J. Plasma Phys., 57, 1-34, 1997.

Bruno, R., Bavassano, B., Pietropaolo, E., Carbone, V., and Veltri, P.: Effects of intermittency on interplanetary velocity and magnetic field fluctuations anisotropy, Geophys. Res. Lett., 26, 3185-3188, 1999.

Buchert, S. C., Fujii, R., and Glassmeier, K.-H.: Ionospheric conductivity modulation in ULF pulsations, J. Geophys. Res., 104, $10119-10133,1999$.

Campbell, W. H.: An analysis of the spectra of geomagnetic variations having periods from $5 \mathrm{~min}$ to 4 hours, J. Geophys. Res., 81, 1369-1390, 1976.

Chang, T.: Low-dimensional behavior and symmetry-breaking of stochastic systems near criticalityCan these effects be observed in space and in the laboratory, IEEE Trans. Plasma Sci., 20, 691694, 1992.

Chapman, S. C., Hnat, B., Rowlands, G., and Watkins, N. W., AScaling collapse and structure functions: identifying self-affinity in finite length time series, Nonlinear Prog. Geophys., 12, 767774, 2005. 
Chisham, G. and Freeman, M. P.: A technique for accurately determining the cusp-region polar cap boundary using SuperDARN HF radar measurements, Ann. Geophys., 21, 983-996, 2003, http://www.ann-geophys.net/21/983/2003/.

Chisham, G. and Freeman, M. P.: An investigation of latitudinal transitions in the SuperDARN Doppler spectral width parameter at different magnetic local times, Ann. Geophys., 22, 1187-1202, 2004 , http://www.ann-geophys.net/22/1187/2004/.

Chisham, G., Freeman, M. P., and Sotirelis, T.: A statistical comparison of SuperDARN spectral width boundaries and DMSP particle precipitation boundaries in the nightside ionosphere, Geophys. Res. Lett., 31, L02804, doi:10.1029/2003GL019074, 2004.

Chisham, G., Freeman, M. P., Sotirelis, T., Greenwald, R. A., Lester, M., and Villain, J.-P.: A statistical comparison of SuperDARN spectral width boundaries and DMSP particle precipitation boundaries in the morning sector ionosphere, Ann. Geophys., 23, 733-743, 2005,

http://www.ann-geophys.net/23/733/2005/.

Chisham, G., Lester, M., Milan, S. E., Freeman, M. P., Bristow, W. A., Grocott, A., McWilliams, K. A., Ruohoniemi, J. M., Yeoman, T. K., Dyson, P. L., Greenwald, R. A., Kikuchi, T., Pinnock, M., Rash, J. P. S., Sato, N., Sofko, G. J., Villain, J.P., and Walker, A. D. M.: A decade of the Super Dual Auroral Radar Network (SuperDARN): Scientific achievements, new techniques and future directions, Surv. Geophys., 28, 33-109, doi:10.1007/s10712-007-9017-8 2007.

Consolini, G., De Michelis, P., Meloni, A., Cafarella, L., and Candidi, M.: Lévy-stable probability distribution of magnetic field fluctuations at Terra Nova Bay (Antarctica), Proc. Italian Res. On Antarctic Atmosphere, 367-376, Soc. Ital. di Fisica, Bologna, Italy, 1998.

Francia, P., Villante, U., and Meloni, A.: An analysis of geomagnetic field variations $(3 \mathrm{~min}-2 \mathrm{~h})$ at a low latitude observatory (L=1.6), Ann. Geophys., 13, 522-531, 1995, http://www.ann-geophys.net/13/522/1995/.

Freeman, M. P., Watkins, N. W., and Riley, D. J.: Evidence for a solar wind origin of the power law burst lifetime distribution of the AE indices, Geophys. Res. Lett., 27, 1087-1090, 2000.

Frisch, U.: Turbulence, Cambridge University Press, Cambridge U.K., 1995.

Greenwald, R. A., Baker, K. B., Dudeney, J. R., Pinnock, M., Jones, T. B., Thomas, E. C., Villain, J.-P., Cerisier, J.-C., Senior, C., Hanuise, C., Hunsucker, R. D., Sofko, G., Koehler, J., Nielsen, E., Pellinen, R., Walker, A. D. M., Sato, N., and Yamagishi, H.: DARN/SuperDARN: A global view of the dynamics of highlatitude convection, Space Sci. Rev., 71, 761-796, 1995.

Grossman, S., Lohse, D., and Reeh, A.: Application of extended self-similarity in turbulence, Phys. Rev. E, 56, 5473-5478, 1997.

Golovchanskaya, I. V., Ostapenko, A. A., and Kozelov, B. V.: Relationship between the high-latitude electric and magnetic turbulence and the Birkeland field-aligned currents, J. Geophys. Res., 111, A12301, doi:10.1029/2006JA011835, 2006.

Hnat, B., Chapman, S. C., Rowlands, G., Watkins, N. W., and Freeman, M. P.: Scaling of solar wind and the AU, AL and AE indices as seen by WIND, Geophys. Res. Lett., 29, 2078, doi:10.1029/2002GL016054, 2002.

Hnat, B., Chapman, S. C., Rowlands, G., Watkins, N. W., and Freeman, M. P.: Scaling in long term data sets of geomagnetic in- dices and solar wind $\epsilon$ as seen by WIND spacecraft, Geophys. Res. Lett., 30, 2174, doi:10.1029/2003GL018209, 2003.

Hnat, B., Chapman, S. C., and Rowlands, G.: Scaling and a FokkerPlanck model for fluctuations in geomagnetic indices and comparison with solar wind $\epsilon$ as seen by Wind and ACE, J. Geophys. Res., 110, A08206, doi:10.1029/2004JA010824, 2005.

Kamide, Y. and Baumjohann, W.: Magnetospher-ionosphere coupling, Springer-Verlag, Berlin Heidelberg, 1993.

Katul, G. G., Parlange, M. B., and Chu, C. R.: Intermittency, local isotropy, and non-Gaussian statistics in atmospheric surfacelayer turbulence, Phys. Fluids, 6, 2480-2492, 1994.

Kintner, P. M.: Observations of velocity shear driven plasma turbulence, J. Geophys. Res., 81, 5114-5122, 1976.

Kozelov, B. V., Uritsky, V. M., and Klimas, A. J.: Power law probability distributions of multiscale auroral dynamics from ground-based TV observations, Geophys. Res. Lett., 31, L20804, doi:10.1029/2004GL020962, 2004.

Krishnamurthy, S., Tanguy, A., Abry, P., and Roux, S.: A stocastic description of external dynamics, Europhys. Lett., 51, 1-7, 2000.

Lui, A. T. Y., Chapman, S. C., Liou, K., Newell, P. T., Meng, C. I., Brittnacher, M., and Parks, G. K.: Is the dynamic magnetosphere an avalanching system?, Geophys. Res. Lett., 27, 911-914, 2000.

Mangeney, A., Salem, C., Veltri, P. L., and Cecconi, B.: Intermittency in the solar wind turbulence and the Haar wavelet transform, in: Sheffield space plasma meeting: multipoint measurement versus theory, Proc. - Les Woolliscroft memorial conference, 53-64, 2001.

Pagel, C., and Balogh, A.: A study of magnetic fluctuations and their anomalous scaling in the solar wind: the Ulysses fastlatitude scan, Nonlinear Prog. Geophys., 8, 313-330, 2001.

Parkinson, M. L.: Dynamical critical scaling of electric field fluctuations in the greater cusp and magnetotail implied by HF radar observations of F-region Doppler velocity, Ann. Geophys., 24, 689-705, 2006, http://www.ann-geophys.net/24/689/2006/.

Pulkkinen, A., Klimas, A., Vassiliadis, D., Uritsky, V., and Tanskanen, E.: Spatiotemporal scaling properties of the ground geomagnetic field variations, J. Geophys. Res., 111, A03305, doi:10.1029/2005JA011294, 2006.

Ruohoniemi, J. M. and Greenwald, R. A.: Dependencies of high-latitude plasma convection: Consideration of interplanetary magnetic field, seasonal, and universal time factors in statistical patterns, J. Geophys. Res., 110, A09204, doi:10.1029/2004JA010815, 2005.

Ruohoniemi, J. M., Greenwald, R. A., Baker, K. B., and Villain, J.-P.: Drift motions of small-scale irregularities in the high latitude $\mathrm{F}$ region: An experimental comparison with plasma drift motions, J. Geophys. Res., 92, 4553-4564, 1987.

Takalo, J., Timonen, J., and Koskinen, H.: Correlation dimension and affinity of AE data and bicolored noise, Geophys. Res. Lett., 25, 1527-1530, 1993.

Takalo, J. and Timonen, J.: Comparison of the dynamics of the AU and PC indices, Geophys. Res. Lett., 25, 2101-2104, 1998.

Taylor, G. I.: The spectrum of turbulence, Proc. R. Soc. London, Ser. A, 164, 476-490, 1938.

Uritsky, V. M., Klimas, A. J., Vassiliadis, D., Chua, D., and Parks, G.: Scale-free statistics of spatiotemporal auroral emissions as depicted by POLAR UVI images: Dynamic magnetosphere is an avalanching system, J. Geophys. Res., 107, 1426 , 
doi:10.1029/2001JA000281, 2002.

Veltri, P. and Mangeney, A.: Scaling laws and intermittent structures in solar wind MHD turbulence, in: Solar Wind 9, AIP Conference Proceedings, 471, 543-546, 1999.

Villain, J.-P., Caudal, G., and Hanuise, C.: A SAFARI-EISCAT comparison between the velocity of $\mathrm{F}$ region small-scale irregularities and the ion drift, J. Geophys. Res., 90, 8433-8443, 1985.
Weatherwax, A. T., Rosenberg, J. T., Lanzerotti, L. J., Maclennan, C. G., Frey, H. U., and Mende, S. B.: The distention of the magnetosphere on 11 May 1999: High latitude Antarctic observations and comparisons with low latitude magnetic and geopotential data, Geophys. Res. Lett., 27, 4029-4032, 2000.

Weimer, D. R., Goertz, C. K., Gurnet, D. A., Maynard, N. C., and Burch, J. L.: Auroral zone electric fields from DE1 and 2 at magnetic conjunction, J. Geophys. Res., 90, 7479-7494, 1985. 